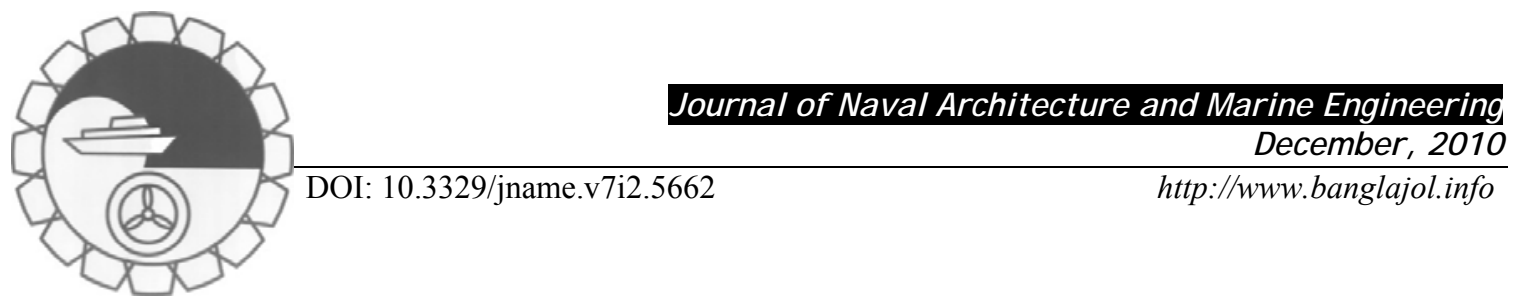

\title{
INDUCED MAGNETIC FIELD WTH RADIATING FLUID OVER A POROUS VERTICAL PLATE: ANALYTICAL STUDY
}

\section{Sahin Ahmed}

Fluid Mechanics Research, Department of Mathematics, Goalpara College, Goalpara 783101, Assam, INDIA, Email: heat_mass@yahoo.in

\begin{abstract}
:
The objective of this investigation is to study the influence of thermal radiation and magnetic Prandtl number on the steady MHD heat and mass transfer by mixed convection flow of a viscous, incompressible, electrically-conducting, Newtonian fluid which is an optically thin gray gas over a vertical porous plate taking into account the induced magnetic field. The similarity solutions of the transformed dimensionless governing equations are obtained by series solution. It is found that, velocity is reduced considerably with a rise in conduction-radiation parameter $(\boldsymbol{R})$ or Hartmann number $(\boldsymbol{M})$ whereas the skin friction is found to be markedly boosted with an increase in $\boldsymbol{M}$ or Magnetic Prandtl number $\left(\boldsymbol{P r}_{\boldsymbol{m}}\right)$. An increase in magnetic body parameter (M) or Magnetic Prandtl number $\left(\boldsymbol{P} \boldsymbol{r}_{\boldsymbol{m}}\right)$ is found to escalate induced magnetic field whereas an increase in $\boldsymbol{R}$ is shown to exert the opposite effect. The acquired knowledge in our study can be used by designers to control Magnetohydrodynamic (MHD) flow as suitable for a certain application. Applications of the study include laminar magneto-aerodynamics, materials processing and MHD propulsion thermo-fluid dynamics.
\end{abstract}

Keywords: Thermal Radiation, Magnetic materials processing, Mixed Convection, Thermal plasma reactors, MHD induction heating, Boundary layers and Magnetic Prandtl number.

\section{NOMENCLATURE}

$\overrightarrow{\boldsymbol{q}} \quad$ The fluid velocity vector,

$\overrightarrow{\boldsymbol{H}} \quad$ The magnetic induction vector,

$H_{0} \quad$ Externally applied transverse magnetic field,

$\bar{H}_{x} \quad$ Induced magnetic field along x-direction,

$\bar{C} \quad$ Species concentration $(\mathrm{kg} . \mathrm{m})$,

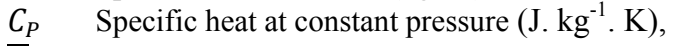

$\bar{C}_{\infty} \quad$ Species concentration in the free stream $\left(\mathrm{kg} . \mathrm{m}^{-3}\right)$,

$\bar{C}_{w} \quad$ Species concentration at the surface $\left(\mathrm{kg} . \mathrm{m}^{-3}\right)$,

$D \quad$ Chemical molecular diffusivity $\left(\mathrm{m}^{2} \cdot \mathrm{s}^{-1}\right)$,

$g$ Acceleration due to gravity (m. $\mathrm{s}^{-2}$ ),

Gr Thermal Grashof number,

$\mathrm{Gm}$ Mass Grashof number,

$M$ Hartmann number/Magnetic parameter,

a Absorption coefficient,

$\mathrm{Pr}_{m}$ Magnetic Prandtl number,

$\operatorname{Pr} \quad$ Prandtl number,

$\bar{\sigma} \quad$ Stefan-Boltzmann constant,

Sc Schmidt number,

$\bar{T} \quad$ Temperature (K),

$\bar{T}_{w} \quad$ Fluid temperature at the surface $(\mathrm{K})$,

$\bar{T}_{\infty} \quad$ Fluid temperature in the free stream $(\mathrm{K})$,

$u \quad$ Velocity component in $\mathrm{x}$-direction $\left(\mathrm{m} . \mathrm{s}^{-1}\right)$

$U_{0}$ Dimensionless free stream velocity $\left(\mathrm{m} . \mathrm{s}^{-1}\right)$, $v_{0} \quad$ Suction velocity $\left(\mathrm{m} . \mathrm{s}^{-1}\right)$,

$\vec{J} \quad$ Current density $\left(\mathrm{m}^{-2}\right.$. s. A),

$q_{r} \quad$ Radiative heat flux,

\section{Greek symbols}

$\beta$ Coefficient of volume expansion for heat transfer $\left(\mathrm{K}^{-1}\right)$,

$\bar{\beta} \quad$ Coefficient of volume expansion for mass transfer $\left(\mathrm{K}^{-1}\right)$,

$\eta \quad$ Magnetic diffusivity,

$\mu_{e} \quad$ Magnetic permeability,

$\mu \quad$ Viscosity of fluid,

$\theta$ Dimensionless fluid temperature,

$\kappa$ Thermal conductivity (W. $\mathrm{m}^{-1} \cdot \mathrm{K}^{-1}$ ),

$v \quad$ Kinematic viscosity $\left(\mathrm{m}^{2} \cdot \mathrm{s}^{-1}\right)$,

$\rho$ Density (kg. m),

$\sigma \quad$ Electrical conductivity,

$\tau \quad$ Shearing stress (N. $\mathrm{m}^{-2}$ ),

$\phi$ Dimensionless species concentration,

\section{Subscripts}

w Conditions on the wall

$\infty$ Free stream conditions 


\section{Introduction}

In the present report, the specific problem selected for study is mixed convection flow involving coupled heat and mass transfer in an electrically conducting fluid adjacent to an isothermal porous plate, with radiation heat transfer effects accounted for. There is an interesting aspect involving MHD effects in forced convection boundary layers; induced magnetic forces modify the free stream flow and this, in turn, affects the external pressure gradient or the free stream velocity that is imposed in the boundary layer. Thus, a complete boundary layer solution would involve a MHD solution for the inviscid free stream. As a consequence, the forced convection problem is more complicated than free convection.

Magneto-hydrodynamics (MHD) is the branch of continuum mechanics which deals with the flow of electrically conducting fluids in electric and magnetic fields. Many natural phenomena and engineering problems are worth being subjected to an MHD analysis. Magneto-hydrodynamic equations are ordinary electromagnetic and hydrodynamic equations modified to take into account the interaction between the motion of the fluid and the electromagnetic field. The formulation of the electromagnetic theory in a mathematical form is known as Maxwell's equation. The effect of the gravity field is always present in forced flow heat transfer as a result of the buoyancy forces connected with the temperature differences. Usually they are of a small order of magnitude so that the external forces may be neglected. There has recently been a considerable interest in the effect of body forces on forced convection phenomena. In certain engineering problems, however, they cannot be left out of consideration. It is important to realize that the heat transfer in mixed convection can be significantly different from that both in pure natural convection and in pure forced convection. The study of forced and free convection flow and heat transfer for electrically conducting fluids past a semi-infinite porous plate under the influence of a magnetic field has attracted the interest of many investigators in view of its applications in many engineering problems such as geophysics, astrophysics, boundary layer control in the field of aerodynamics. Engineers employ MHD principle, in the design of heat exchangers pumps and flow meters, in space vehicle propulsion, thermal protection, braking, control and re-entry, in creating novel power generating systems etc.

The radiation effects have important applications in physics and engineering, particularly in space technology and high temperature processes. But very little is known about the effects of radiation on the boundary layer. Thermal radiation effects on the boundary layer may play important role in controlling heat transfer in polymer processing industry where the quality of the final product depends on the heat controlling factors to some extent. High temperature plasmas, cooling of nuclear reactors, liquid metal fluids, power generation systems are some important applications of radiative heat transfer. Actually, many processes in new engineering areas occur at high temperatures and knowledge of radiation heat transfer beside the convective heat transfer becomes very important for the design of the pertinent equipment. Nuclear power plants, gas turbines and the various propulsion devices for aircraft, missiles, satellites and space vehicles are examples of such engineering areas. Moreover, when radiative heat transfer takes place, the fluid involved can be electrically conducting since it is ionized due to the high operating temperature. Accordingly, it is of interest to examine the effect of the magnetic field on the flow. Studying such effect has great importance in the application fields where thermal radiation and MHD are correlative. In all these applications understanding the behaviour of MHD free and forced convective flow and the various problem parameters that influence is a very important asset to designers developing applications that aim to control this flow. For example, the process of fusing of metals in an electrical furnace by applying a magnetic field and the process of cooling of the first wall inside a nuclear reactor containment vessel where the hot plasma is isolated from the wall by applying a magnetic field.

Over the past years, this problem attracted the attention of several researchers. However, none of them included all relevant aspects that influence the flow behaviour. A summary of researcher's efforts is as follows: Merkin (1969) investigated the mixed convection boundary layer flow on a semi-infinite vertical flat plate when the buoyancy forces aid and oppose the development of boundary layer. Watanabe (1991) presented a laminar forced and free mixed convection flow on a flat plate with uniform suction or injection was theoretically investigated. Non-similar partial differential equations are transformed into non-similar ordinary ones by means of difference-differential method. Kafousias et al. (1979) considered the effects of free convection currents on the flow field of an incompressible viscous fluid past an impulsively started infinite vertical porous limiting surface when the fluid is subjected to suction with uniform velocity using the Laplace transform technique. Recently, the study of heat and mass transfer on the free convective flow of a viscous incompressible fluid past an infinite vertical porous plate in presence of transverse sinusoidal suction velocity and a constant free stream velocity was presented by Ahmed (2009). Also, Ahmed and Liu (2010) analyzed the effects of mixed convection and mass transfer of three-dimensional oscillatory flow of a viscous incompressible fluid past an infinite vertical porous plate in presence of transverse sinusoidal suction velocity oscillating with time and a constant free stream velocity. The non-linear coupled equations of the works $(2009,2010)$ were solved analytically by employing perturbation technique. 
Soundalgekar (1965) investigated the Hydromagnetic flow of a viscous incompressible fluid due to uniformly accelerated motion of an infinite flat plate in the presence of a magnetic field fixed relative to the plate and he found that velocity at any point and at any instant decreases when the strength of the magnetic field is increased. Kafousias and Georgantopoulos (1982) studied the transverse magnetic effects on the free convective flow of an incompressible, electrically conducting fluid past a non-conducting and non-magnetic, vertical limiting surface, the governing equations were solved by the usual Laplace transform technique. Raptis and Soundalgekar (1984) determined the effects of mass transfer on the flow of an electrically conducting fluid past a steadily moving infinite vertical porous plate under the action of a transverse magnetic field. Hussain et al. (2000) considered the problem of natural convection boundary layer flow, induced by the combined buoyancy forces from mass and thermal diffusion from a permeable vertical flat surface with non-uniform surface temperature and concentration but a uniform rate of suction of fluid through the permeable surface. Abdelkhalek (2009) presented for heat and mass transfer effect on hydromagnetic flow of a moving permeable vertical surface. The non-linear coupled boundary layer equations were transformed and the resulting ordinary differential equations were solved by perturbation technique.

The above studies have generally been confined to very small magnetic Reynolds numbers, allowing magnetic induction effects to be neglected. Such effects must be considered for relatively large values of the magnetic Reynolds number. Glauert (1962) presented a seminal analysis for hydromagnetic flat plate boundary layers along a magnetized plate with uniform magnetic field in the stream direction at the plate. He obtained series expansion solutions (for both large and small values of the electrical conductivity parameter) for the velocity and magnetic fields, indicating that for a critical value of applied magnetic field, boundary-layer separation arise. Raptis and Soundalgekar (1982) considered the problem of flow of an electrically conducting fluid past a steadily moving vertical infinite plate in presence of constant heat flux and constant suction at the plate and induced magnetic field is also taken into account. Recently, Bég et al. (2009) obtained local non-similarity numerical solutions for the velocity, temperature and induced magnetic field distributions in forced convection hydromagnetic boundary layers, over an extensive range of magnetic Prandtl numbers and Hartmann numbers. Alom et al. (2008) investigated the steady MHD heat and mass transfer by mixed convection flow from a moving vertical porous plate with induced magnetic, thermal diffusion, constant heat and mass fluxes and the non-linear coupled equations are solved by shooting iteration technique.

England and Emery (1969) have studied the radiation effects of an optically thin gray gas bounded by a stationary plate. Raptis and Massalas (1998) investigated the effects of radiation on the oscillatory flow of a gray gas, absorbing-emitting in presence induced magnetic field and analytical solutions were obtained with help of perturbation technique. They found out that the mean velocity decreases with the Hartmann number, while the mean temperature decreases as the radiation increases. The hydrodynamic free convective flow of an optically thin gray gas in the presence of radiation, when the induced magnetic field is taken into account was studied by Raptis et al. (2003) using perturbation technique. They concluded that the velocity and induced magnetic field increase as the radiation increases. Hossain et al. (1999) determined the effect of radiation on the natural convection flow of an optically dense incompressible fluid along a uniformly heated vertical plate with a uniform suction. The governing non-similar boundary-layer equations are analyzed using (i) a series solution; (ii) an asymptotic solution; and (iii) a full numerical solution. Magnetohydrodynamic mixed free-forced heat and mass convective steady incompressible laminar boundary layer flow of a gray optically thick electrically conducting viscous fluid past a semi-infinite vertical plate for high temperature and concentration differences have studied by Emad and Gamal (2005). Orhan and Kaya (2008) investigated the mixed convection heat transfer about a permeable vertical plate in the presence of magneto and thermal radiation effects using the Keller box scheme, an efficient and accurate finite-difference scheme. They concluded that, an increase in the radiation parameter decreases the local skin friction parameter and increases the local heat transfer parameter. Ghosh et al. (2009) considered an exact solution for the hydromagnetic natural convection boundary layer flow past an infinite vertical flat plate under the influence of a transverse magnetic field with magnetic induction effects and the transformed ordinary differential equations are solved exactly.

As the importance of radiation in the fields of aerodynamics as well as space science technology, the present study is motivated towards this direction. The main objective of the present investigation will, therefore, be to study the effects of radiation and magnetic Prandtl number on the steady mixed convective heat and mass transfer flow of an optically thin gray gas over an infinite vertical porous plate with constant suction in presence of transverse magnetic field, by means of analytical solutions. These analytical approximate solutions under perturbation technique give a wider applicability in understanding the basic physics and chemistry of the problem, which are particularly important in industrial and technological fields. The present analysis is valid for the case of a permeable vertical wall. 


\section{Mathematical Model}

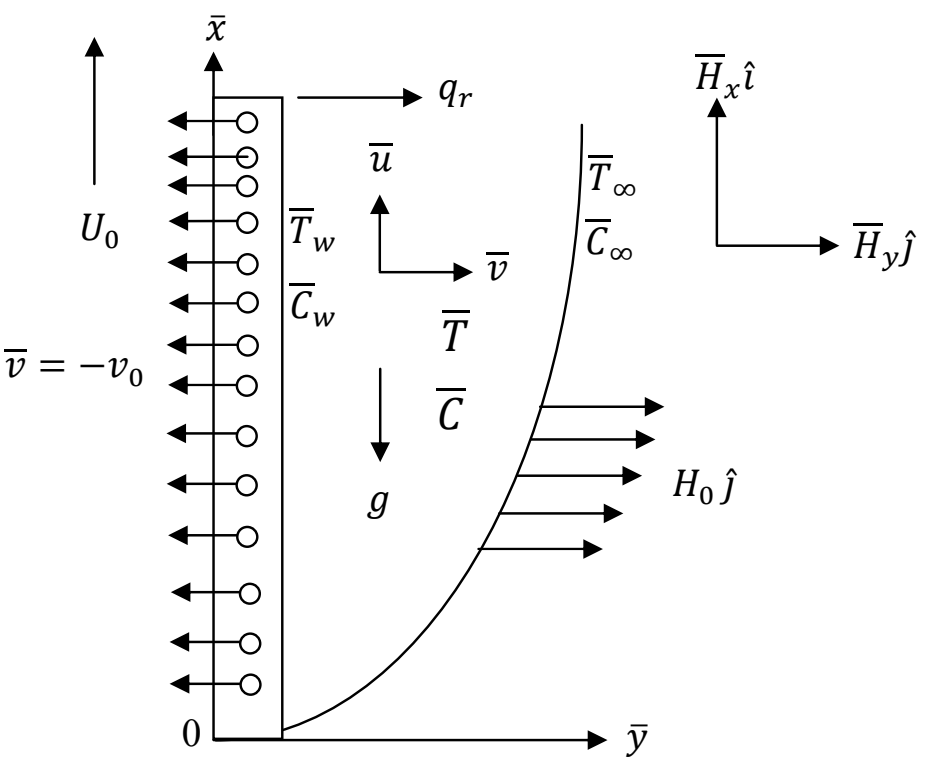

Figure 1: Physical configuration and coordinate system

The two-dimensional steady magnetohydrodynamic mixed convective heat and mass transfer flow of a Newtonian, electrically-conducting and viscous incompressible fluid over a porous vertical infinite plate with induced magnetic field and conduction-radiation has been considered in Figure 1. Exact solutions of equations are obtained by perturbation technique. The following assumptions are implicit in our analysis:

- All the fluid properties except the density in the buoyancy force term are constant;

- The Eckert number, $E c$, is small, as appropriate for viscous incompressible regimes;

- The plate is subjected to a constant suction velocity;

- The plate is non-conducting and the applied magnetic field is of uniform strength $\left(H_{0}\right)$ and applied transversely to the direction of the main stream taking into account the induced magnetic field;

- The magnetic Reynolds number of the flow is not taken to be small enough so that the induced magnetic field is not negligible;

- The concentration of the diffusing species in the binary mixture is assumed to be very small in comparison with the other chemical species, which are present, and hence the Soret and Dufour effects are negligible;

- The equation of conservation of electric charge is $\nabla . J=0$, where $J=\left(J_{x}, J_{y}, J_{z}\right)$.

- The direction of propagation is considered only along the y-axis and does not have any variation along the $\mathrm{y}$-axis and so $\frac{\partial J_{y}}{\partial y}=0$, which gives $J_{y}=$ constant. Since the plate is electrically non-conducting, this constant is zero and hence $J_{y}=0$ everywhere in the flow, following Sutton and Sherman (1965).

- The wall is maintained at constant temperature $\bar{T}_{w}$ and concentration $\bar{C}_{w}$ higher than the ambient temperature $\bar{T}_{\infty}$ and concentration $\bar{C}_{\infty}$ respectively.

- The fluid is non-magnetic, neglecting the thermoelectric effect as well as viscous and electrical dissipation together with the short circuit condition.

Let $\overrightarrow{\boldsymbol{q}}=(\overline{\boldsymbol{u}}(\boldsymbol{y}), \overline{\boldsymbol{v}}, \mathbf{0})$ be the fluid velocity and $\overrightarrow{\boldsymbol{H}}=\left(\overline{\boldsymbol{H}}_{\boldsymbol{x}}(\boldsymbol{y}), \overline{\boldsymbol{H}}_{\boldsymbol{y}}, \mathbf{0}\right)$ be the magnetic induction vector at a point $(\bar{x}, \bar{y}, \bar{z})$ in the fluid. The $\overline{\boldsymbol{x}}$-axis is taken along the plate in the upward direction, $\overline{\boldsymbol{y}}$-axis is normal to the plate into the fluid region. Since the plate is infinite in length in $\bar{x}$-direction, therefore all the physical quantities except possibly the pressure are assumed to be independent of $\bar{x}$.

For a steady-state incompressible magnetohydrodynamic mixed convection flow and mass transfer with radiation, given by Sherman and Sutton (1965), and Maxwell's equations, the fundamental equations are:

$\operatorname{div} \overrightarrow{\boldsymbol{q}}=0$, Conservation of Mass,

$\operatorname{div} \overrightarrow{\boldsymbol{H}}=0$, Gauss's law of magnetism, 
$\rho(\overrightarrow{\boldsymbol{q}} . \boldsymbol{\nabla}) \overrightarrow{\boldsymbol{q}}=-\boldsymbol{\nabla} p+\mu \boldsymbol{\nabla}^{2} \overrightarrow{\boldsymbol{q}}+\mu_{e}(\overrightarrow{\boldsymbol{J}} X \overrightarrow{\boldsymbol{H}})+\rho \overrightarrow{\boldsymbol{g}}$, Conservation of Momentum,

$(\overrightarrow{\boldsymbol{q}} \cdot \boldsymbol{\nabla}) \bar{T}=\frac{1}{\rho C_{P}}\left(\kappa \nabla^{2} \bar{T}-\frac{\partial q_{r}}{\partial \bar{y}}\right)$, Conservation of Energy,

$\boldsymbol{\nabla} X(\overrightarrow{\boldsymbol{q}} X \overrightarrow{\boldsymbol{H}})+\eta \boldsymbol{\nabla}^{2} \overrightarrow{\boldsymbol{H}}=0$, Conservation of Magnetic Induction,

$(\overrightarrow{\boldsymbol{q}} \cdot \boldsymbol{\nabla}) \bar{C}=D \nabla^{2} \bar{C}$, Conservation of species,

$\nabla X \overrightarrow{\boldsymbol{H}}=\overrightarrow{\boldsymbol{J}}$

All the physical quantities are defined in the Nomenclature.

With the foregoing assumptions and under the usual boundary layer and Boussinesq approximations, Equations (1) to (6) reduce to:

$\frac{\partial \bar{v}}{\partial \bar{y}}=0$ which is satisfied with $\bar{v}=-v_{0}=$ a constant.

$\frac{\partial \bar{H}_{y}}{\partial \bar{y}}=0$ which holds for $\bar{H}_{y}=H_{0}=$ a constant $=$ strength from applied magnetic field

$\rho \bar{v} \frac{\partial \bar{u}}{\partial \bar{y}}=-\frac{\partial p}{\partial \bar{x}}-\rho g+\mu \frac{\partial^{2} \bar{u}}{\partial \bar{y}^{2}}+\mu_{e} H_{0} \frac{\partial \bar{H}_{x}}{\partial \bar{y}}$

$\bar{v} \frac{\partial \bar{T}}{\partial \bar{y}}=\frac{\kappa}{\rho C_{P}} \frac{\partial^{2} \bar{T}}{\partial \bar{y}^{2}}-\frac{1}{\rho C_{P}} \frac{\partial q_{r}}{\partial \bar{y}}$

$\bar{v} \frac{\partial \bar{H}_{x}}{\partial \bar{y}}=\frac{1}{\sigma \mu_{e}} \frac{\partial^{2} \bar{H}_{x}}{\partial \bar{y}^{2}}+H_{0} \frac{\partial \bar{u}}{\partial \bar{y}}$

$\bar{v} \frac{\partial \bar{C}}{\partial \bar{y}}=D \frac{\partial^{2} \bar{C}}{\partial \bar{y}^{2}}$

Since there is no large velocity gradient here, the viscous term in Equation (10) vanishes for small $\mu$ and hence for the outer flow, beside there is no induced magnetic field along $\mathrm{x}$-direction gradient, so we have

$0=-\frac{\partial p}{\partial \bar{x}}-\rho_{\infty} g$

By eliminating the pressure term from Equations (10) and (14), we obtain

$\rho \bar{v} \frac{\partial \bar{u}}{\partial \bar{y}}=\left(\rho_{\infty}-\rho\right) g+\mu \frac{\partial^{2} \bar{u}}{\partial \bar{y}^{2}}+\mu_{e} H_{0} \frac{\partial \bar{H}_{x}}{\partial \bar{y}}$

The Boussinesq approximation gives

$\rho_{\infty}-\rho=\rho_{\infty} \beta\left(\bar{T}-\bar{T}_{\infty}\right)+\rho_{\infty} \bar{\beta}\left(\bar{C}-\bar{C}_{\infty}\right)$

On using (16) in the equation (15) and noting that $\rho_{\infty}$ is approximately equal to 1 , the momentum equation reduces to

$-v_{0} \frac{\partial \bar{u}}{\partial \bar{y}}=g \beta\left(\bar{T}-\bar{T}_{\infty}\right)+g \bar{\beta}\left(\bar{C}-\bar{C}_{\infty}\right)+v \frac{\partial^{2} \bar{u}}{\partial \bar{y}^{2}}+\frac{\mu_{e} H_{0}}{\rho} \frac{d \bar{b}_{x}}{d \bar{y}}$

The boundary conditions are:

$\left.\begin{array}{l}\bar{y}=0: \bar{u}=0, \bar{v}=-v_{0}, \bar{T}=\bar{T}_{w}, \bar{H}_{x}=0, \bar{C}=\bar{C}_{w} \\ \bar{y} \rightarrow \infty: \bar{u} \rightarrow U_{0}, \bar{T} \rightarrow \bar{T}_{\infty}, \bar{H}_{x} \rightarrow 0, \bar{C} \rightarrow \bar{C}_{\infty}\end{array}\right\}$

The non-dimensional quantities are: 
$y=\frac{v_{0} \bar{y}}{v}, \quad u=\frac{\bar{u}}{U_{0}}, \theta=\frac{\bar{T}-\bar{T}_{\infty}}{\bar{T}_{w}-\bar{T}_{\infty}}, \phi=\frac{\bar{c}-\bar{C}_{\infty}}{\bar{C}_{w}-\bar{C}_{\infty}}, S c=\frac{v}{D}, \quad \operatorname{Pr}=\frac{\rho v C_{P}}{\kappa}$,

$G r=\frac{v g \beta\left(\bar{T}_{w}-\bar{T}_{\infty}\right)}{U_{0} v_{0}^{2}}, G m=\frac{v g \bar{\beta}\left(\bar{C}_{w}-\bar{C}_{\infty}\right)}{U_{0} v_{0}^{2}}, P r_{m}=\sigma v \mu_{e}$,

$M=\sqrt{\frac{\mu_{e}}{\rho}} \frac{H_{0}}{v_{0}}, B=\sqrt{\frac{\mu_{e}}{\rho}} \frac{\bar{H}_{x}}{U_{0}}, R=\frac{64 a v \bar{\sigma} \bar{T}_{\infty}^{3}}{\rho v_{0}^{2} C_{P}}$

For the case of an optically thin gray gas, the local radiant absorption is expressed as (2003):

$\frac{\partial q_{r}}{\partial \bar{y}}=-4 a \bar{\sigma}\left(\bar{T}_{\infty}^{4}-\bar{T}^{4}\right)$

where ' $a$ ' is the mean absorption coefficient and $\bar{\sigma}$ is the Stefan-Boltzmann constant. It is assumed that the temperature differences within the flow are sufficiently small such that $\bar{T}^{4}$ may be expressed as linear function of the temperature $\bar{T}$. This is accomplished by expanding $\bar{T}^{4}$ in Taylor series about $\bar{T}_{\infty}$ and neglecting higherorder terms $(2003,2000)$, thus

$\bar{T}^{4} \cong 4 \bar{T}_{\infty}^{3} \bar{T}-3 \bar{T}_{\infty}^{4}$

Using the transformations (19) and with help of (20) and (21), the non-dimensional forms of (11), (12), (13) and (17) are

$\frac{d^{2} u}{d y^{2}}+\frac{d u}{d y}+M \frac{d B}{d y}+\operatorname{Gr} \theta+\operatorname{Gm} \phi=0$

$\frac{d^{2} \theta}{d y^{2}}+\operatorname{Pr} \frac{d \theta}{d y}+\frac{\operatorname{Pr} R}{4} \theta=0$

$\frac{d^{2} B}{d y^{2}}+M P r_{m} \frac{d u}{d y}+P r_{m} \frac{d B}{d y}=0$

$\frac{d^{2} \phi}{d y^{2}}+S c \frac{d \phi}{d y}=0$

The corresponding boundary conditions are:

$y=0: u=0, \theta=1, B=0, \phi=1$

$y \rightarrow \infty: u \rightarrow 1, \theta \rightarrow 0, \quad B \rightarrow 0, \phi \rightarrow 0$

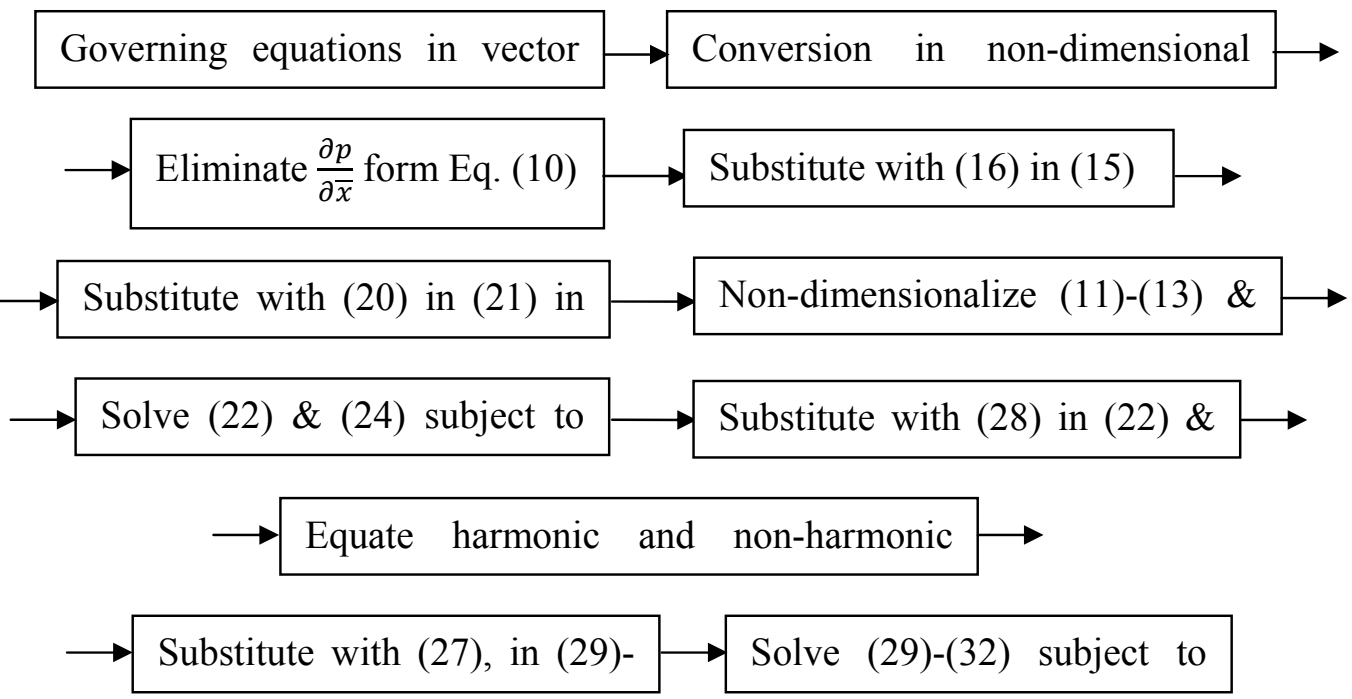

Fig. 2: Flowcharts of the proposed work 


\section{Method of Solution}

Perturbation theory leads to an expression for the desired solution in terms of a power series in some "small" parameter that quantifies the deviation from the exactly solvable problem. The leading term in this power series is the solution of the exactly solvable problem, while further terms describe the deviation in the solution, due to the deviation from the initial problem. Perturbation theory is applicable if the problem at hand can be formulated by adding a "small" term (Eckert number in this work) to the mathematical description of the exactly solvable problem.

The solutions of the equations (23) and (25) subject to the boundary condition (26) are

$\theta=e^{-\xi y}, \phi=e^{-S c y}$

where $\xi=\frac{\left[P r+\sqrt{P r^{2}+R P r}\right]}{2}$.

Now, in order to solve the equations (22) and (24) under the boundary condition (26), we note that $E c \ll 1$ (Eckert number) for all the incompressible fluids and it is assumed the solutions of the equations to be of the form

$\Re(y)=\Re_{0}(y)+E c \Re_{1}(y)+O\left(E c^{2}\right)$,

In which $\mathfrak{R}$ stands for $u$ or $B$, and $\Re_{0}$ is the mean part and $\Re_{1}$ is the perturbed part. Substituting (28) into the equations (22) and (24) and equating the coefficients of the same degree terms and neglecting terms of $+O\left(E c^{2}\right)$, the following ordinary differential equations are obtained, in which ()$^{\prime}$ designates $d / d y$ :

$u_{0}^{\prime \prime}+u_{0}^{\prime}=-G r \theta-G m \phi-M B_{0}^{\prime}$

$u_{1}^{\prime \prime}+u_{1}^{\prime}=-M B_{1}^{\prime}$

$B_{0}^{\prime \prime}+P r_{m} B_{0}^{\prime}=-M P r_{m} u_{0}^{\prime}$

$B_{1}^{\prime \prime}+P r_{m} B_{1}^{\prime}=-M P r_{m} u_{1}^{\prime}$

The boundary conditions (26) reduce to

$y=0: u_{0}=0, u_{1}=0, B_{0}=0, B_{1}=0$

$y \rightarrow \infty: u_{0} \rightarrow 1, u_{1} \rightarrow 0, B_{0} \rightarrow 0, B_{1} \rightarrow 0$

The solutions of the velocity and induced magnetic field subject to the boundary conditions (33) are:

$u(y)=1+A_{1} \exp (-\xi y)+A_{2} \exp (-S c y)+A_{3} \exp (-\lambda y)$

$B(y)=A_{4} \exp (-\xi y)+A_{5} \exp (-S c y)+A_{6} \exp (-\lambda y)+A_{7} \exp \left(-P r_{m} y\right)$

where

$$
\begin{aligned}
& \lambda=\frac{\left[1+P r_{m}+\sqrt{\left(1-P r_{m}\right)^{2}+4 M^{2} P r_{m}}\right]}{2}, A_{1}=\frac{G r\left(\xi-P r_{m}\right)}{-\xi^{3}+\left(1+P r_{m}\right) \xi^{2}+\xi\left(M^{2}-1\right) P r_{m}}, \\
& A_{2}=\frac{G m\left(\eta-P r_{m}\right)}{-S c^{3}+\left(1+P r_{m}\right) S c^{2}+S c\left(M^{2}-1\right) P r_{m}}, A_{3}=-\left(1+A_{1}+A_{2}\right), A_{4}=\frac{M A_{1} P r_{m}}{\xi-P r_{m}}, \\
& A_{5}=\frac{M A_{2} P r_{m}}{S c-P r_{m}}, A_{6}=\frac{M A_{3} P r_{m}}{\lambda-P r_{m}}, A_{7}=-\left(A_{4}+A_{5}+A_{6}\right) .
\end{aligned}
$$

\section{Skin-Friction}

The boundary layer produces a drag on the plate due to the viscous stresses which are developed at the wall. The viscous stress at the surface of the plate is given by

$\tau=\left(\frac{\partial u}{\partial y}\right)_{y=0}=-\xi \mathrm{A}_{1}-S c \mathrm{~A}_{2}-\lambda A_{3}$

\section{Results and discussion}

To assess the effects of the dimensionless thermophysical parameters on the regime, we have carried out the calculations for the velocity field, induced magnetic field and electric current density at the plate. The results are presented graphically in figures 3 to 10 . All data is provided in each figure. 
Figure 3 illustrate the velocity response for magnetic field $(M)$ and Prandtl number $(P r)$ due to cooling of the plate $(G r>0)$ i.e. free convection currents convey heat away from the plate in to the boundary layer. With an increase in $M$ from the non-conducting i.e. purely hydrodynamic case $(M=0)$ through 0.50 to 0.75 , there is a strong deceleration in the flow is achieved. The presence of a magnetic field in an electrically-conducting fluid introduces a force called Lorentz force which acts against the flow if the magnetic field is applied in the normal direction as considered in the present problem. This type of resistive force tends to slow down the flow field. Since the magnetic field has a stabilizing effect, the maximum velocity overshoot is observed for the conducting air, while minimum overshoot takes place for the water. Moreover, there is clear decrease in velocity values at the wall accompanying a rise in $\operatorname{Pr}$ from 0.71 (conducting air) to 7.0 (water) i.e. the flow is decelerated. Higher $\mathrm{Pr}$ fluids will therefore posses higher viscosities (and lower thermal conductivities) implying that such fluids will flow slower than lower $\operatorname{Pr}$ fluids. As a result the velocity will be decreased substantially with increasing Prandtl number.

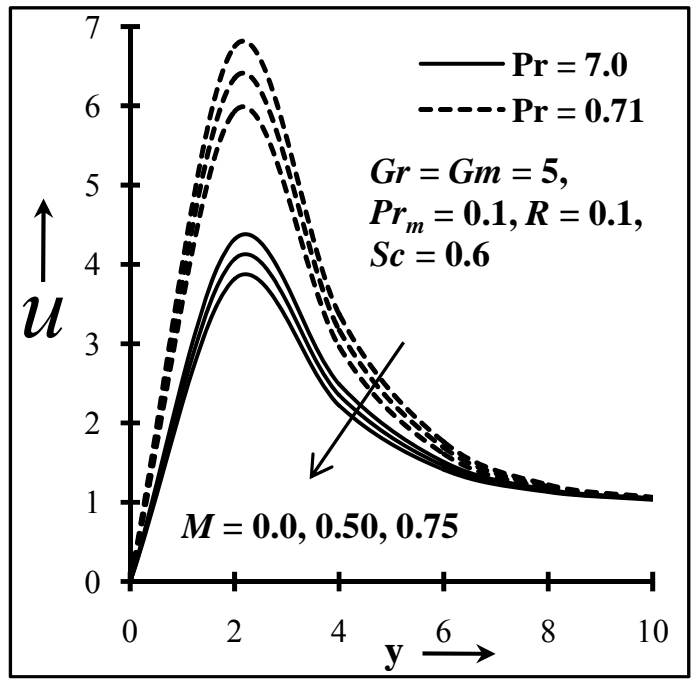

Fig. 3: Velocity distribution for $M$ and $P r$

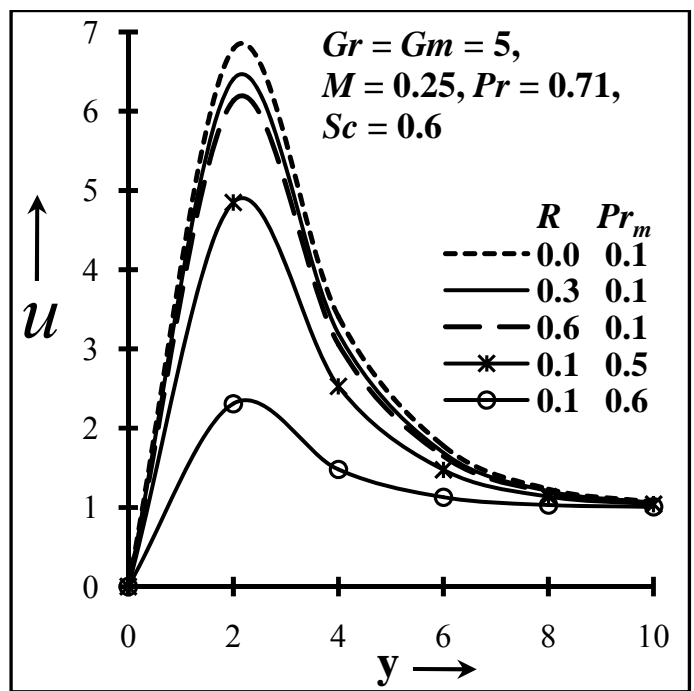

Fig. 4: Velocity distribution for $R$ and $P r_{m}$

Fig. 4 shows the influence of the radiation parameter $(R)$ and magnetic Prandtl number $\left(P r_{m}\right)$ on the velocity field $(u)$ in presence of conducting air $(P r=0.71)$ and weak magnetohydrodynamic flow $(M=0.25)$. The velocity remains positive for all values of $R$ and $P r_{m}$ i.e. there is no flow reversal within the boundary layer. With an increase in $R$ from 0.0 (non-radiating) through 0.3 to 0.7 (thermal conduction is dominant over radiation), there is a clear decrease in velocity i.e. the flow is decelerated. This may be attributed to the fact that the increase in $R$ implies less interaction of radiation with the momentum boundary layer. Moreover, a rise in $\mathrm{Pr}_{m}$ value from 0.1 through 0.5 to 0.6 (in all these cases magnetic diffusion rate exceeds the viscous diffusion rate) causes a noticeable decreasing in the flow velocity, in particular at short distance from the wall.

The effects of Hartmann number $(M)$ and Prandtl number $(P r)$ on the induced magnetic field, $B$ has been presented in Figure 5 in presence of weak magnetohydrodynamic flow $(M=0.25)$ and Oxygen $(S c=0.60$, diffusing in air, $\operatorname{Pr}=0.71$ ). For all combinations of $M$ and $P r$, values of $H$ are remains negative i.e. induced magnetic flux reversal arises for all distances into the boundary layer, transverse to the plate. In all the cases, $H$ values peak a short distance from the plate; profiles thereafter decay to the zero value in the free stream. For $\mathrm{Pr}$ $=0.71$, the $H$ values become increasingly negative i.e. greater flux reversal arises in the boundary layer when $M=0.75$; but for $\operatorname{Pr}=7.0$, the induced magnetic is found to less negative when $M=0.25$. Therefore, when $\mathrm{Pr}$ increases from 0.71 to 7.0 , the induced magnetic field is found to decrease absolutely. Moreover, a rise in $M$ from 0.25 through 0.50 to 0.75 serves to elevated the induced magnetic field magnitudes throughout the regime for both cases of $\operatorname{Pr}=0.71$ and $\operatorname{Pr}=7.0$.

The effects of radiation parameter $(R)$ and magnetic Prandtl number $\left(P r_{m}\right)$ on the induced magnetic field $(B)$ are presented in the respective Figures 6 and 7. In this figure magnetic Prandtl number, $P r_{m}$ is set as less than unity, which implies that the magnetic diffusion rate exceeds the viscous diffusion rate. As such Pm increases, momentum diffusivity will be increased. Therefore, when $P r_{m}$ increases from 0.1 to 0.4 , the induced magnetic field is found to increase absolutely in the boundary layer $0 \leq y<6$, but this trend is opposite for the region $6<y \leq 10$. Greater flux reversal arises in the boundary layer region $y \in\left[0,6\left[\right.\right.$ and for $P r_{m}=0.4$ (magnetic diffusion rate exceeds the viscous diffusion rate); but this trend is reversed for the region $y \in] 6,10]$. However, a rise in $R$ from 0.0 through $0.3,0.9$ to 1.5 depresses the induced magnetic field magnitudes 
throughout the regime. It is seen that, the influence of $R$ and $P r_{m}$ is very important near the wall as well as very significantly far away from the wall.

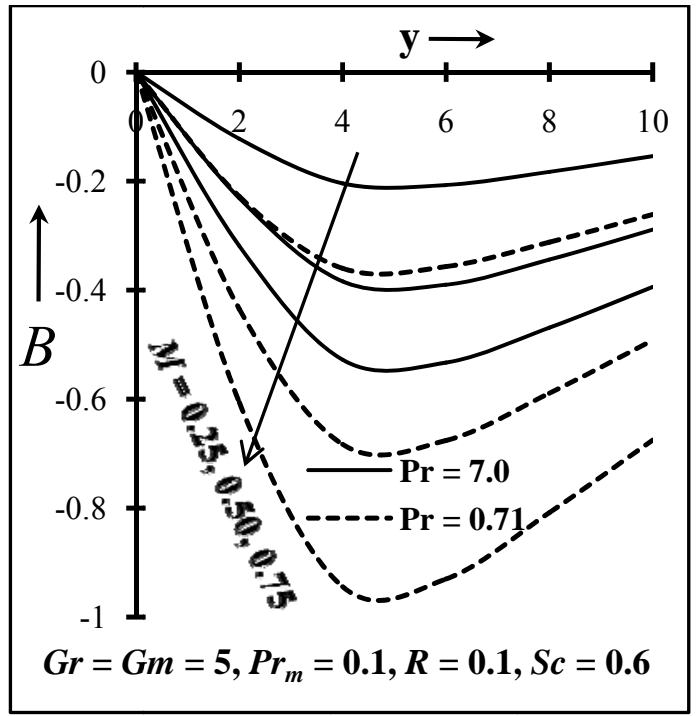

Fig. 5: Induced magnetic field for $M$ and $P r_{m}$

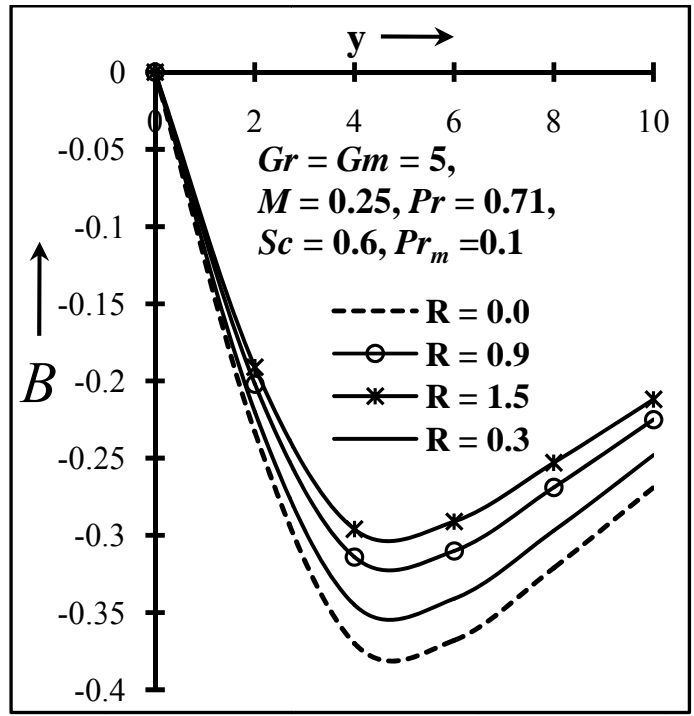

Fig. 6: Induced magnetic field for $R$

In Figure 8 the response of fluid temperature $(\theta)$ to the radiation parameter $(R)$ is plotted for $\operatorname{Pr}=0.71$ (physically conducting air). Increasing radiation parameter from 0.0 (non-radiating) through $0.6,1.5$ to 2.5 clearly depressed the fluid temperature. The radiation parameter arises only in the energy equation (11) in the thermal diffusion term, $\frac{1}{\rho C_{P}}\left[\kappa \frac{\partial^{2} \bar{T}}{\partial \bar{y}^{2}}-\frac{\partial q_{r}}{\partial \bar{y}}\right]$, and via coupling of the temperature field $(\bar{T})$ with the buoyancy terms in the momentum equation (17), the velocity field is indirectly influenced by thermal radiation effects. For $R=1$ thermal radiation and thermal conduction contributions are equivalent. For $R>1$ thermal radiation is dominant over conduction and vice versa for $R<1$.

Figure 9 depicts the flow velocity $(u)$ with various Schmidt number $(S c)$ due to cooling of the plate $(G r>0)$. An increase in Sc from 0.66 (Oxygen) through 0.78 (Ammonia) to 0.94 (Carbon dioxide) (in all these cases species diffusivity rate exceeds momentum diffusivity rate), will suppress flow velocity in the boundary layer regime i.e. the flow is decelerated.

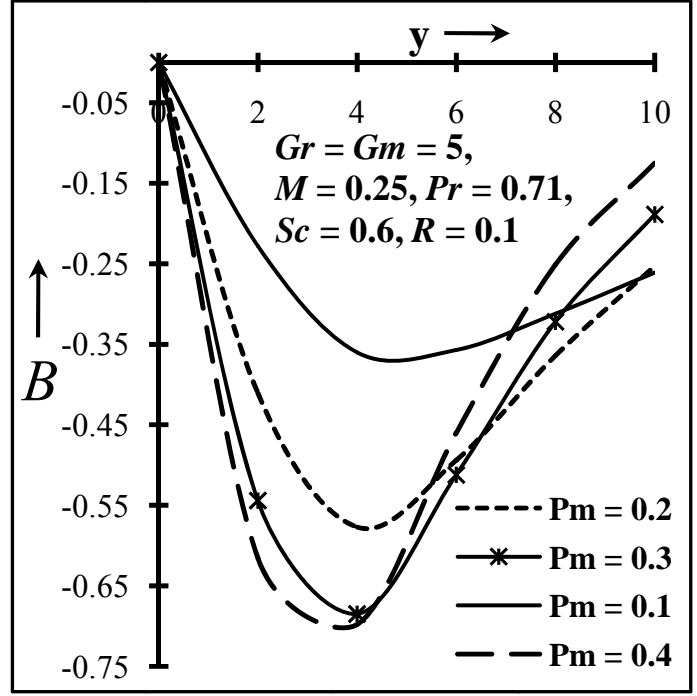

Fig. 7: Induced magnetic field for $P r_{m}$

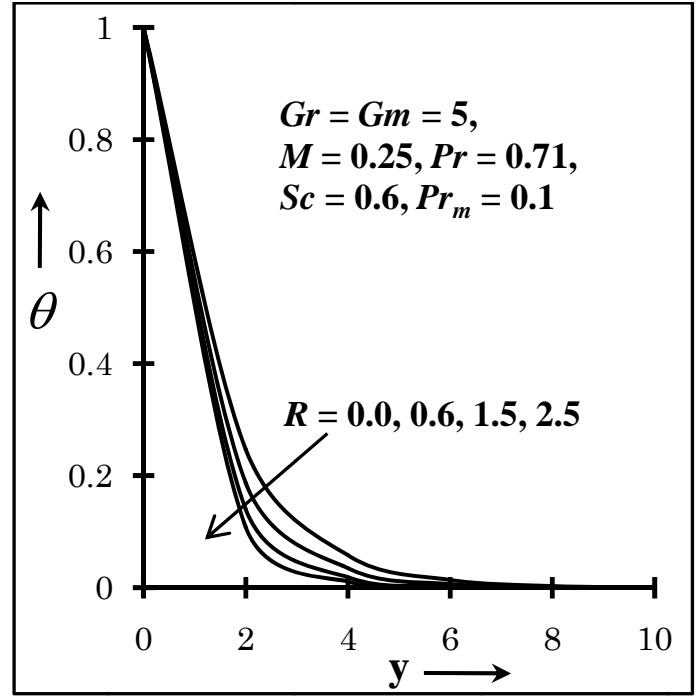

Fig. 8: Temperature field for $R$

The effects of Hartmann number $(M)$ and magnetic Prandtl number $\left(P r_{m}\right)$ on the skin friction $(\tau)$ are shown in Fig. 10 for the conducting air $(\operatorname{Pr}=0.71)$. The magnitude of skin-friction at the plate increases linearly due to the increase in $M$ and $P r_{m}$. 
Sahin Ahmed / Journal of Naval Architecture and Marine Engineering 7(2010) 83-94

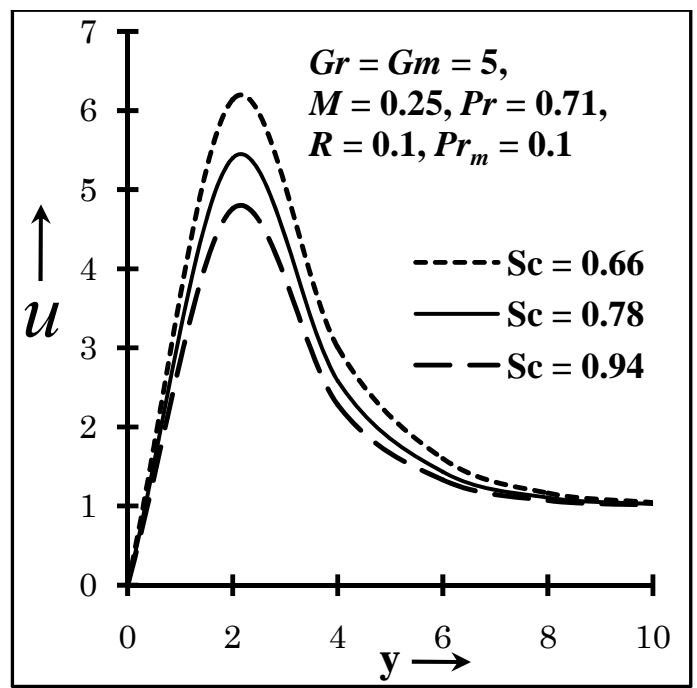

Fig. 9: Velocity field for $S c$

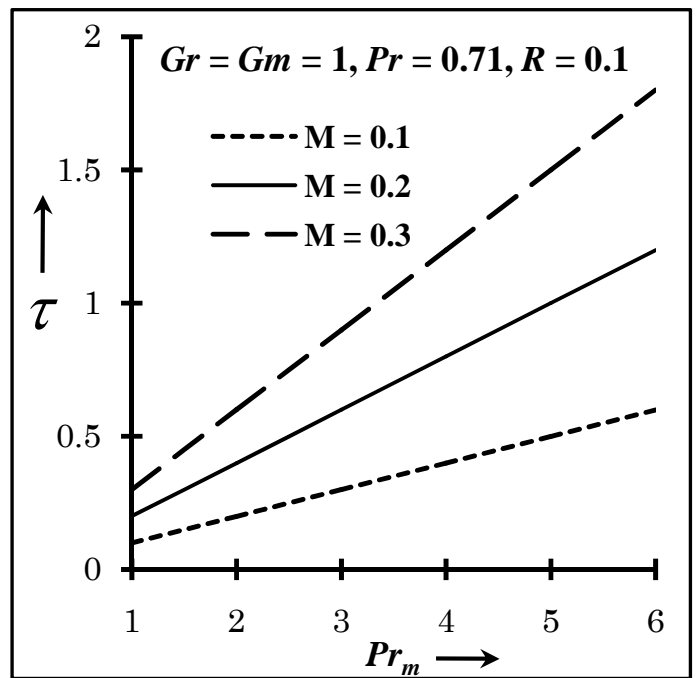

Fig. 10: Skin friction for $M$ and $P r_{m}$

The method is validated by directly comparing its results with those of Raptis et al. (2003) with $M=$ $0.25, G m=0.0, G r=5$ as shown in Table 1 and 2, where $P m=0.1<1$ which implies that magnetic diffusion rate is dominant over the viscous diffusion rate. From Tables 1 and 2, it is seen that both the results for flow velocity and induced magnetic field are in excellent agreement. Therefore, this leads to confidence in the numerical results. In Table 1 , it is observed that the flow velocity is decelerated when conduction-radiation increases from 0.1 through 0.8 to 1.0 . In Table 2 , a rise in $R$ from 0.1 through 0.8 to 1.0 serves to depress the induced magnetic field magnitudes throughout the regime. These results are similar to the results in Figs. 3 and 5 .

Table 1: Comparison of values of the flow velocity $(u)$ with those obtained by Raptis et al. (2003) for Pm= $0.1<M=0.25, G m=0.0$ and $G r=5$ :

\begin{tabular}{|c|l|l|l|l|l|l|}
\hline & \multicolumn{3}{|c|}{ Raptis et al. (2003) } & \multicolumn{3}{c|}{ Present work } \\
\hline $\mathrm{y}$ & $R=0.1$ & \multicolumn{1}{|c|}{$R=0.8$} & $R=1.0$ & $R=0.1$ & $R=0.8$ & \multicolumn{1}{|c|}{$R=1.0$} \\
\hline 0.0 & 0.0 & 0.0 & 0.0 & 0.0 & 0.0 & 0.0 \\
\hline 2.0 & 3.39344 & 2.83302 & 2.77543 & 3.35839 & 2.70542 & 2.68540 \\
\hline 4.0 & 1.88766 & 1.57742 & 1.53862 & 1.85232 & 1.51786 & 1.46272 \\
\hline 6.0 & 1.24757 & 1.13381 & 1.11986 & 1.24063 & 1.12212 & 1.10496 \\
\hline 8.0 & 1.06255 & 1.02759 & 1.02367 & 1.06134 & 1.02555 & 1.02107 \\
\hline 10.0 & 1.01511 & 1.00536 & 1.00439 & 1.01492 & 1.00503 & 1.00396 \\
\hline
\end{tabular}

Table 2: Comparison of values of the induced magnetic field $(B)$ with those obtained by Raptis et al. (2003) for $P m=0.1<M=0.25, G m=0.0$ and $G r=5$ :

\begin{tabular}{|c|l|l|l|l|l|l|}
\hline & \multicolumn{3}{|c|}{ Raptis et al. (2003) } & \multicolumn{3}{c|}{ Present work } \\
\hline $\mathrm{y}$ & \multicolumn{1}{|c|}{$R=0.1$} & $R=0.8$ & $R=1.0$ & $R=0.1$ & $R=0.8$ & $R=1.0$ \\
\hline 0.0 & 0.0 & 0.0 & 0.0 & 0.0 & 0.0 & 0.0 \\
\hline 2.0 & -0.09437 & -0.07526 & -0.07392 & -0.08750 & -0.06367 & -0.05916 \\
\hline 4.0 & -0.14786 & -0.11214 & -0.10870 & -0.13880 & -0.09687 & -0.08914 \\
\hline 6.0 & -0.14358 & -0.10543 & -0.10150 & -.013538 & -0.09161 & -0.08389 \\
\hline 8.0 & -0.12354 & -0.08931 & -0.08594 & -0.11669 & -0.07775 & -0.07101 \\
\hline 10.0 & -0.10263 & -0.7472 & -0.07070 & -0.09699 & -0.06421 & -0.05859 \\
\hline
\end{tabular}




\section{Conclusion:}

This study presents a theoretical treatment of steady magnetohydrodynamic boundary layer flow and combined heat and mass transfer of an incompressible, electrically-conducting and radiating fluid over an infinite vertical permeable plate, taking into account the magnetic Prandtl number. The observations are:

- An increase in radiation parameter/Hartmann number leads to decelerate the flow velocity, while increasing Hartmann number elevated the Induced magnetic field.

- Increasing conduction-radiation acts to depress flow velocity and induced magnetic field.

- It was also observed that increasing magnetic Prandtl number effects elevated the induced magnetic field near the plate, while this trend is reversed away from the plate.

- Velocity is reduced considerably with a rise in Schmidt number or Prandtl number.

- Temperature is reduced by the increase of radiation.

- Skin friction is strongly elevated by the increase of Hartmann number or magnetic Prandtl number.

- Using magnetic field we can control the flow characteristics and heat transfer.

- Radiation has significant effects on the velocity as well as temperature distributions.

The present study has been confined to Newtonian viscous model. Future investigations will consider viscoelastic and power-law rheological fluid models and will be communicated in the near future.

\section{References:}

Merkin, J. H. (1969): The effects of buoyancy forces on the boundary-layer flow over a semi-infinite vertical flat plate in a uniform stream, Journal of Fluid Mechanics, Vol. 35, No. 3, pp. 439-450.

Watanabe, T. (1991): Forced and free mixed convection boundary layer flow with uniform suction or injection on a vertical flat plate, Acta Mechanica, Vol. 89, No. 1-4, pp. 123-132. doi: 10.1007/BF01171250

Kafousias, N. G., Nanousis, N. and Georgantopoulos, G. A. (1979): Free convection effects on the Stokes problem for an infinite vertical limiting surface with constant suction, Astrophysics and Space Science, Vol. 64, pp. 391-399. doi: 10.1007/BF00639516

Ahmed, S. (2009): The study of heat and mass transfer on free convective three-dimensional unsteady flows over a porous vertical plate, Journal of Energy, Heat and Mass Transfer, Vol. 31, pp. 89-110.

Ahmed, S. and Liu, I-Chung (2010): Mixed convective three-dimensional heat and mass transfer flow with transversely periodic suction velocity, Int. J. Applied Mathematics and Mechanics, Vol. 6, pp. 58-73.

Soundalgekar, V. M. (1965): Hydromagnetic flow near an accelerated plate in the presence of a magnetic field, Applied Scientific Research, Vol. 12, No. 1, pp. 151-156.

Kafousias, N. G. and Georgantopoulos, G. A. (1982): Magnetohydrodynamic free convection effects on the Stokes problem for an incompressible viscous fluid past an infinite vertical limiting surface, Astrophysics and Space Science, Vol. 85, No. 1-2, pp. 297-307. doi: 10.1007/BF00653451

Raptis, A. and Soundalgekar, V. M. (1984): MHD flow past a steadily moving infinite vertical porous plate with mass transfer and constant heat flux, ZAMM - Journal of Applied Mathematics and Mechanics, Vol. 64, No. 2, pp. 127-130. doi: 10.1002/zamm.19840640208

Hussain, S., Hossain, M. A. and Wilson, M. (2000): Natural convection flow from a vertical permeable flat plate with variable surface temperature and species concentration, Engineering Computations, Vol. 17, No. 7, pp. 789-812. doi: $10.1108 / 02644400010352261$

Abdelkhalek, M. M. (2009): Heat and mass transfer in MHD free convection from a moving permeable vertical surface by a perturbation technique, Communications in Nonlinear Science and Numerical Simulation, Vol. 14, No. 5, pp. 2091-2102. doi: 10.1016/j.cnsns.2008.06.001

Glauert, M. B. (1962): The boundary layer on a magnetized plate, J. Fluid Mechanics, Vol. 12, No. 4, pp. 625638.

Raptis, A. A. and Soundalgekar, V. M. (1982): MHD flow past a steadily moving infinite vertical porous plate with constant heat flux, Nuclear Engineering and Design, Vol. 72, No. 3, pp. 373-379. doi:10.1016/0029$\underline{5493(82) 90050-4}$

Induced magnetic field with radiating fluid over a porous vertical plate: Analytical study 
Bég, O. A., Bakier, A. Y., Prasad, V. R., Zueco, J. and Ghosh, S. K. (2009): Nonsimilar, laminar, steady, electrically-conducting forced convection liquid metal boundary layer flow with induced magnetic field effects, Int. J. Thermal Sciences, Vol. 48, pp. 1596-1606. doi:10.1016/j.ijthermalsci.2008.12.007

Alom, M. M., Rafiqul, I. M. and Rahman, F. (2008): Steady heat and mass transfer by mixed convection flow from a vertical porous plate with induced magnetic field, constant heat and mass fluxes, Thammasat Int. J. Sc. Tech., Vol. 13, No. 4, pp. 1-13. doi:10.1155/DENM/2006/32675

England, W. G. and Emery, A. F. (1969): Thermal radiation effects on the laminar free convection boundary layer of an absorbing gas, J. of Heat Transfer, Vol. 91, pp. 37-44.

Raptis, A., and Massalas, C. V. (1998): Magnetohydrodynamic flow past a plate by the presence of radiation, Heat and Mass transfer, Vol. 34, pp. 107-109. doi: 10.1007/s002310050237

Raptis, A. A., Perdikis, C. and Leontitsis, A. (2003): Effects of radiation in an optically thin gray gas flowing past a vertical infinite plate in the presence of a magnetic field, Heat and mass Transfer, Vol. 39, pp. 771-773. doi: $10.1007 / \mathrm{s} 00231-002-0317-8$

Hossain, M. A., Alim, M. A. and Rees, D. A. S. (1999): The effect of radiation on free convection from a porous vertical plate, Int. J. Heat and Mass Transfer, Vol. 42, No. 1, pp. 181-191. doi:10.1016/S0017-9310(98)00097-0

Emad M. A. and Gamal El-Din A. A. (2005): Thermal radiation effects on magnetohydrodynamic flow past a semi-infinite vertical plate in the presence of mass diffusion, Can. J. Phys., Vol. 83, No. 3. Pp. 243-256. doi: 10.1139/P04-084

Orhan A. and Ahmet K. (2008): Radiation effect on MHD mixed convection flow about a permeable vertical plate, Heat Mass Transfer, Vol. 45, pp. 239-246. doi 10.1007/s00231-008-0428-y,m

S. K. Ghosh, Bég, O. A. and Zueco, J. (2009): Hydromagnetic free convection flow with induced magnetic field effects, Physics and Astronomy, Volume 45, Number 2, 175-185.

Sutton, G. W. and Sherman, A. (1965): Engineering magnetohydrodynamics, Macgraw-hill, New york 